\title{
Port Free Chemotherapy for Recurrent or Metastatic Colorectal Cancer. Is Port Really Necessary?
}

\section{Yoichiro Yoshida*}

Department of Gastroenterological Surgery, Fukuoka University Faculty of Medicine, 7-45-1 Nanakuma, Jonan-ku, Fukuoka 814-0180, Japan

Keywords: Colorectal cancer; Chemotherapy; Central venous port; Vascular pain; Oxaliplatin

\section{Background}

The introduction of totally implantable port systems started in the early 1980s [1]. Today, these devices provide easy vascular access for delivery of chemotherapy, fluids, medications, blood products and parenteral nutrition solutions. Over the last few decades, many management changes in oncology have occurred, particularly with respect to new chemotherapy combinations and more complex application schemes. Cancer patients usually require repeated venous punctures for treatment monitoring, application of chemotherapy or blood transfusions. Central venous catheters and implantable port systems have therefore substantially facilitated the problem of vascular access. To date, safe and easy-to-handle port systems have become an integral part of daily clinical routine in oncology [2]. However, there are several important complications associated with central venous (CV) port [3]. After immediate perioperative and short-term complications such as accidental arterial puncture, hematoma, air embolism, pneumothorax or vessel perforation [4], clinical oncologists are most often concerned with major long-term complications occurring during the use of ports in daily routine care. Chemotherapy for colorectal cancer has advanced remarkably with the introduction of folinic acid, fluorouracil, and irinotecan (FOLFIRI); and folinic acid, fluorouracil, and oxaliplatin (FOLFOX); therapies, which require a $\mathrm{CV}$ port. With the recent development of capecitabine plus oxaliplatin (XELOX) therapy, capecitabine plus irinotecan (XELIRI) therapy and irinotecan plus S-1 (IRIS) therapy involving oral administration of drug preparations, etc. Implantation of a CV port can be now avoided.

However, vascular pain occasionally requires switching of the drip infusion route during XELOX therapy by the administration of oxaliplatin via the peripheral vein. Vascular pain and phlebitis induced by intravenous infusion of antineoplastic agents reduces the completion or continuation of chemotherapy. The causative factors of vascular pain and phlebitis include the $\mathrm{pH}$ and osmotic pressure of the solution, size of the vein used, size and material of the catheter, and infusion periods [5]. A number of methods for avoiding phlebitis have been reported [6-7]; however, none of them are completely effective. Thus, there is an urgent need to develop new methods to prevent and alleviate phlebitis. Some investigators reported that addition of steroids to oxaliplatin drip infusion is useful in controlling vascular pain [8]. However, the pharmacological use of steroids can make oxaliplatin unstable due to the elevation of $\mathrm{pH}$; further, the effectiveness of oxaliplatin in this therapy is unknown because of lack of published data in this regard. However, we recently reported that the effectiveness of dexamethasone (DEX) for controlling vascular pain caused by the administration of oxaliplatin via the peripheral vein during XELOX therapy [9].
Furthermore, we found that co-infusion of DEX to oxaliplatin may be a useful preventive method for oxaliplatin-induced hypersensitivity. We may be able to Port Free Chemotherapy for colorectal cancer by making full use of XELOX, XELIRI and IRIS therapy involving oral administration of drug preparations. However, further studies will be needed to determine the effectiveness of this method.

\section{References}

1. Niederhuber JE, Ensminger W, Gyves JW, Liepman M, Doan K, et al. (1982) Totally implanted venous and arterial access system to replace external catheters in cancer treatment. Surgery 92: 706-712.

2. Torramadé JR, Cienfuegos JA, Hernández JL, Pardo F, Benito C, et al. (1993) The complications of central venous access systems: a study of 218 patients. Eur J Surg 159: 323-327.

3. Mansfield PF, Hohn DC, Fornage BD, Gregurich MA, Ota DM (1994) Complications and failures of subclavian-vein catheterization. $\mathrm{N}$ Engl J Med 331: 1735-1738.

4. Teichgräber UK, Gebauer B, Benter T, Wagner J (2004) Long-term central venous lines and their complications. Rofo 176: 944-992.

5. Kuwahara T, Asanami S, Kubo S (1998) Experimental infusion phlebitis: tolerance osmolality of peripheral venous endothelial cells. Nutrition 14: 496501.

6. Nakayama S, Matsubara N, Sakai T, Aso N (2002) The incidence of phlebitis in the patients administrated vinorelbine by intravenous bolus injection--a retrospective study. Gan To Kagaku Ryoho 29: 633-635.

7. Curran CF, Luce JK, Page JA (1990) Doxorubicin-associated flare reactions. Oncol Nurs Forum 17: 387-389.

8. Matsuyama K, Mishima H, Ueno H, Kajihara K, Morioka A, et al. (2011) Etiology and management of venous pain during intravenous administration of oxaliplatin. Gan To Kagaku Ryoho 38: 411-414.

9. Yoshida Y, Hoshino S, Aisu N, Shiwaku H, Beppu R, et al. (2012) Dexamethasone as a means not only for controlling vascular pain caused by the administration of oxalioplatin via the peripheral vein but also for controlling oxaliplatin-induced hypersensitivity reactions. Br J Med Med Res (in press).

\footnotetext{
*Corresponding author: Yoichiro Yoshida, Department of Gastroenterological Surgery, Fukuoka University, Faculty of Medicine, 7-45-1 Nanakuma, Jonan-ku, Fukuoka 814-0180, Japan, Tel: +81-92-801-1011; Fax: +81-92-863-9759; E-mail: yy4160@yahoo.co.jp

Received February 08, 2012; Accepted February 13, 2012; Published February 15,2012

Citation: Yoshida Y (2012) Port Free Chemotherapy for Recurrent or Metastatic Colorectal Cancer. Is Port Really Necessary? J Cancer Sci Ther 4: iv-iv. doi:10.4172/1948-5956.1000e105

Copyright: @ 2012 Yoshida Y. This is an open-access article distributed under the terms of the Creative Commons Attribution License, which permits unrestricted use, distribution, and reproduction in any medium, provided the original author and source are credited.
} 